\title{
Cushing's syndrome: a practical approach to diagnosis and differential diagnoses
}

\author{
Joseph M Pappachan, ${ }^{1}$ Christian Hariman, ${ }^{1}$ Mahamood Edavalath, ${ }^{2}$ Julian Waldron, ${ }^{3}$ \\ Fahmy W Hanna ${ }^{4}$
}

'Department of Endocrinology \& Diabetes, New Cross Hospital The Royal Wolverhampton Hospitals NHS Trust, Wolverhampton, UK 2Department of Endocrinology \& Diabetes, Imperial College London Diabetes Centre, Abu Dhabi, UAE

${ }^{3}$ Department of Clinical Biochemistry, University Hospitals of North Midlands NHS Trust, Stoke-on-Trent, UK ${ }^{4}$ Department of Endocrinology \& Diabetes, University Hospitals of North Midlands NHS Trust \& North Staffordshire University, Stoke-on-Trent, UK

\section{Correspondence to}

Dr Joseph M Pappachan, Department of Endocrinology \& Diabetes, New Cross Hospital, The Royal Wolverhampton Hospitals NHS Trust,

Wolverhampton WV10 0QP, UK; drpappachan@yahoo.co.in

Received 9 August 2016 Revised 1 November 2016 Accepted 8 December 2016 Published Online First

9 January 2017
CrossMark

To cite: Pappachan JM, Hariman C, Edavalath M, et al. J Clin Pathol 2017:70:350-359.

\section{ABSTRACT}

Diagnosis of Cushing's syndrome (CS) and identification of the aetiology of hypercortisolism can be challenging. The Endocrine Society clinical practice guidelines recommends one of the four tests for initial screening of CS, namely, urinary-free cortisol, late night salivary cortisol, overnight dexamethasone suppression test or a longer low-dose dexamethasone suppression test, for 48 hours. Confirmation and localisation of CS requires additional biochemical and radiological tests. Radiological evaluation involves different imaging modalities including MRI with or without different radionuclear imaging techniques. Invasive testing such as bilateral inferior petrosal sinus sampling may be necessary in some patients for accurate localisation of the cause for hypercortisolism. This best practice review discusses a practical approach for the diagnostic evaluation of CS with a brief discussion on differential diagnoses, and cyclical CS, to enhance the skills of clinicians and laboratory personnel.

\section{INTRODUCTION}

Cushing's syndrome (CS) is the state of hypercortisolism that results from endogenous or exogenous glucocorticoid excess. It is associated with increased morbidity and mortality from musculoskeletal, metabolic, thrombotic, infectious and cardiovascular complications. ${ }^{1}{ }^{2}$ Patients with CS present with protean clinical and biochemical manifestations depending on the aetiology, duration, severity of the disease and physical characteristics of the patient, making the diagnostic work-up a challenge to physicians, radiologists and clinical chemists alike.

The clinical manifestations of CS can vary widely from mild or non-specific symptoms, which are difficult to recognise, to florid features of profound cortisol excess, which can be easily identified. ${ }^{1}{ }^{3}$ Because of the diversity and non-specific nature of the clinical presentation, diagnosis may be often delayed and patients may present at a late stage of the disease or with a complication such as an osteoporotic fracture.

A majority of cases of CS results from adrenocorticotropin (ACTH)-secreting pituitary adenomas that cause overproduction of cortisol from adrenal cortex (termed Cushing's disease (CD)). Sometimes ectopic production of ACTH or corticotrophinreleasing hormone $(\mathrm{CRH})$ from different extrapituitary tumours causes CS. Primary adrenal diseases with cortisol overproduction may also result in CS in some cases (termed adrenal Cushing's).

CS is known to cause increased morbidity and mortality. ${ }^{4}$ Therefore, prompt clinical suspicion, early diagnostic work-up and management are necessary to avoid potential adverse outcomes. This best practice article compiles an evidence-based practical approach to empower clinicians and laboratory personnel for diagnostic evaluation of $\mathrm{CS}$ with the most up-to-date scientific literature.

\section{CLINICAL SUSPICION OF CS}

Patients with classical signs may present with weight gain/obesity, plethora, hypertension, hirsutism, ecchymosis, striae, lethargy, mental health problems, menstrual irregularities, decreased libido and proximal myopathy. ${ }^{3}$ Clinical features encountered less commonly are headache, backache, oedema, abdominal pain, acne, recurrent infections, female baldness, dorsal fat pad, frank diabetes, electrocardiographic abnormalities suggestive of cardiac hypertrophy, osteopenia/osteoporotic fractures and cardiovascular disease from accelerated atherosclerosis. Therefore, patients may be referred to different specialists depending on their clinical presentation (eg, orthopaedic surgeon for fracture, gynaecologist for menstrual problems, dermatologist for skin changes and psychiatrist for mental health issues).

Although some of the clinical features may be sensitive for CS diagnosis, they may not be specific for the disease. Some patients may present with fluctuating symptoms and signs due to a rhythmic variation in cortisol secretion that results in a state of cyclical CS. ${ }^{6}{ }^{7}$ Phenotypic features of CS may occur from overactivity (physiological) of the hypothalamic-pituitary-adrenal (HPA) axis in conditions such as chronic alcoholism, psychiatric disorders, severe obesity, poorly controlled diabetes and states of extreme physical stress. This condition is referred to as pseudo-Cushing's syndrome (PCS). ${ }^{8}$ Subclinical Cushing's syndrome (SCS), seen in some cases of adrenal incidentalomas, is a result of alteration in the HPA axis without overt signs or symptoms of hypercortisolism. SCS presents with biochemical evidence of cortisol excess without the classical phenotypic abnormalities. ${ }^{9} 10$

Broder et $a l^{11}$ recently demonstrated 10 key conditions observed in patients with CS that may help easy identification of patients with CD from those without the disease. The relative risk (RR) of any one of these conditions (selected by expert opinion), viz. localised adiposity, hirsutism, facial plethora, polycystic ovary syndrome, abnormal weight gain, hypokalaemia, deep venous thrombosis, muscle weakness, female balding and osteoporosis, was $\geq 5$ times in $\mathrm{CD}$ cases compared with non-CD cases. Varying combinations of these conditions in dyads or with other associated clinical 
conditions showed higher RR in cases that may help clinicians to easily identify probable patients with $\mathrm{CD}$ as shown in table 1 . An important limitation of this study is that the findings are based on expert opinion without proper biochemical backup of those cases with CD diagnosis.

The possibility of iatrogenic (exogenous) CS should be excluded while evaluating a patient with suspected CS. A comprehensive history and review of all medications including food supplements and even use of over-the-counter topical creams is mandatory in all the cases. This is very important because in some patients the history of exogenous steroid use causing CS may be vague and can mislead the clinician to perform unnecessary diagnostic evaluation. The negative feedback inhibition from chronic exogenous steroid use causes suppression of the ACTH from pituitary and CRH from hypothalamus, with gradual atrophy of the adrenal glands. ${ }^{12}$

\section{INITIAL SCREENING TESTS: EVIDENCES, SENSITIVITY, SPECIFICITY AND CONFOUNDING FACTORS}

The American Endocrine Society Clinical Guidelines (2008) recommends one of the four following tests for the initial screening of CS: two measurements of urinary-free cortisol (UFC), two measurements of late night salivary cortisol (LNSC),
$1 \mathrm{mg}$ overnight dexamethasone suppression test (ODST) or a longer low-dose dexamethasone suppression test (LDDST) with $2 \mathrm{mg} /$ day in divided doses for 48 hours. ${ }^{13}$ The decision regarding suitability of the appropriate initial screening test should be made by the clinician, the patient and the local availability of the tests. Conventionally, UFC and ODST are the preferred tests by most centres/clinicians.

\section{UFC measurement}

This test is performed with a 24-hour collection of urine on at least two separate occasions. This measures the unbound cortisol filtered by renal system, which provides an integrated assessment of total urinary cortisol excretion over a 24-hour period, unlike measurement of (total) serum cortisol (measuring both free hormone and cortisol, which is bound to cortisol binding globulin (CBG)), which may vary alongside alterations in CBG levels in disease states or the concomitant use of different drugs. ${ }^{13}$ To improve the sensitivity of UFC, the upper limit of normal laboratory range should be used as the cut-off value. ${ }^{1} 101314$ Although Ceccato et al ${ }^{15}$ reported a sensitivity of $97 \%$ and specificity of $91 \%$ for UFC levels $>170 \mathrm{nmol} /$ 24 hours using mass spectroscopic method, a more recent study by Aranda et $a l^{16}$ using similar laboratory methods showed

Table 1 Relative risk (RR) in patients with versus without Cushing's disease (CD) of dyads of conditions selected by clinical content expert opinion

\begin{tabular}{|c|c|c|c|c|c|c|}
\hline \multirow[b]{2}{*}{ Order } & \multirow[b]{2}{*}{ Condition 1} & \multirow[b]{2}{*}{ Condition 2} & \multirow{2}{*}{$\begin{array}{l}\text { Development } \\
\text { data set } \\
\text { RR }\end{array}$} & \multicolumn{3}{|c|}{ Validation data set } \\
\hline & & & & $\begin{array}{l}\text { CD } \\
\text { Frequency (\%) }\end{array}$ & $\begin{array}{l}\text { Non-CD } \\
\text { Frequency (\%) }\end{array}$ & $\mathbf{R R}$ \\
\hline 1 & Hypertension & Hirsutism & 70.0 & $49(2.613)$ & $0(0.000)$ & $\infty$ \\
\hline 2 & Serious infections & Adrenal mass & $\infty$ & $42(2.240)$ & $0(0.000)$ & $\infty$ \\
\hline 3 & Type 2 diabetes & Hirsutism & 62.0 & $30(1.600)$ & $0(0.000)$ & $\infty$ \\
\hline 4 & Uncontrolled type 2 diabetes & Premature menopause & 44.0 & $15(0.800)$ & $0(0.000)$ & $\infty$ \\
\hline 5 & Weakness/fatigue & Hirsutism & 35.0 & $64(3.413)$ & $1(0.027)$ & 128.0 \\
\hline 6 & Hyperlipidaemia & Adrenal mass & $\infty$ & $56(2.987)$ & $1(0027)$ & 112.0 \\
\hline 7 & Type 2 diabetes & Adrenal mass & $\infty$ & $52(2.773)$ & $1(0.027)$ & 104.0 \\
\hline 8 & Psychiatric disorders & Hirsutism & 43.0 & $49(2.613)$ & $1(0.027)$ & 98.0 \\
\hline 9 & Serious infections & Hirsutism & $\infty$ & $44(2.347)$ & $1(0.027)$ & 88.0 \\
\hline 10 & Sleep disorders & Adrenal mass & $\infty$ & $35(1.867)$ & $1(0.027)$ & 70.0 \\
\hline 11 & Uncontrolled type 2 diabetes & Hypokalaemia & 15.0 & $32(1.707)$ & $1(0.027)$ & 64.0 \\
\hline 12 & Psychiatric disorders & Adrenal mass & $\infty$ & $63(3.360)$ & $2(0.053)$ & 63.0 \\
\hline 13 & Weakness/fatigue & Adrenal mass & $\infty$ & $56(2.987)$ & $2(0.053)$ & 56.0 \\
\hline 14 & Obesity & Osteoporosis & 10.0 & $26(1.387)$ & $1(0.027)$ & 52.0 \\
\hline 15 & $\begin{array}{l}\text { Metabolic syndrome/impaired } \\
\text { glucose tolerance } \\
\text { /pre-diabetes }\end{array}$ & $\begin{array}{l}\text { Vertebral, long bone, } \\
\text { rib, pelvic and foot } \\
\text { fracture }\end{array}$ & 38.0 & $18(0.960)$ & $1(0.027)$ & 36.0 \\
\hline 16 & Weakness/fatigue & Female balding & 15.3 & $30(1.600)$ & $3(0.080)$ & 20.0 \\
\hline 17 & Type 2 diabetes & Weakness/fatigue & 8.9 & $184(9.813)$ & $22(0.587)$ & 16.7 \\
\hline 18 & Type 2 diabetes & Hypokalaemia & 19.3 & $56(2.987)$ & $7(0.187)$ & 16.0 \\
\hline 19 & Obesity & Weakness/fatigue & 11.7 & $150(8.000)$ & $22(0.587)$ & 13.6 \\
\hline 20 & Hypertension & Osteoporosis & 15.6 & $95(5.067)$ & $19(0.507)$ & 10.0 \\
\hline 21 & Type 2 diabetes & Premature menopause & 31.3 & $35(1.867)$ & 7 (0187) & 10.0 \\
\hline 22 & Osteoporosis & Serious infections & 15.1 & $66(3.520)$ & $16(0.427)$ & 8.3 \\
\hline 23 & Hypertension & Serious infections & 4.6 & 269 (14.347) & $106(2.827)$ & 5.1 \\
\hline 24 & Psychiatric disorders & Serious infections & 4.5 & $245(13.067)$ & $119(3.173)$ & 4.1 \\
\hline
\end{tabular}


much poorer diagnostic utility for UFC levels (UFC $\geq 258.1 \mathrm{nmol} / 24$ hours showed only $53 \%$ sensitivity and $86 \%$ specificity) as an initial screening test for CS. Because of the wide discrepancy in the reported sensitivity and specificity of UFC measurements, many authorities consider this test less useful for initial screening of CS.

Procedural difficulties with the urine collection and processing are the main problems encountered with UFC as a screening tool for CS. Procedure can be cumbersome especially in older patients and small children as the entire urine specimen voided in a 24-hour period should be collected. The test requires a plain 24-hour urine bottle without preservatives and kept refrigerated if not processed immediately. To reduce the likelihood of error, patients should be instructed to avoid excess fluid intake and topical glucocorticoid therapies during the collection period.

UFC levels can be affected by variations in renal function. Urinary cortisol excretion depends on glomerular filtration rate and urinary volume. Therefore, UFC measurement can be erroneously low in patients with renal impairment ${ }^{17}$ and high when urinary volumes exceed $5 \mathrm{~L}$ in 24 hours. ${ }^{18}$ Any physiological or pathological states of cortisol overproduction may also be associated with higher UFC levels. ${ }^{13}$ CS severity has not been shown to be correlated with the level of urinary hypercortisoluria. ${ }^{19-21}$ Up to $15 \%$ of CS cases may be missed by a single measurement of 24-hour UFC. ${ }^{21}$ Although negative 24-hour UFC test in three samples may exclude CS in most cases, in rare situations (mild disease or cyclical CS) the diagnosis may be missed owing to the episodic nature of cortisol hypersecretion. Measurement of urinary cortisol levels on several occasions may be necessary in such situations. To reduce the procedural difficulties with UFC measurement on multiple specimens, calculation of spot urinary cortisol to creatinine ratio with early morning urine samples can be used as an alternative. ${ }^{7}$ Alternative mode of testing may be necessary if only one UFC reading among two or three tests turns positive in patients with clinical suspicion of CS.

There are significant variations in the reported normal ranges in UFC measurement values by different laboratories. This is dependent on the biochemical methods used for cortisol assays. Radioimmunoassay (RIA) and ELISA use antibody-based immunoassays (IAs). Cross-reacting cortisol metabolites and synthetic glucocorticoid molecules may interfere with result analysis and interpretation. ${ }^{13}$ Newer modalities and methodologies such as high-performance liquid chromatography (HPLC), gas chromatography mass spectrometry and liquid chromatography-tandem mass spectrometry (LC-MS/MS) are structurally based methods without these inherent problems and will minimise the variability.

An additional confounder is the concomitant use of medications such as carbamazepine and fenofibrate that may impose interference with some of the biochemical assays, ${ }^{13} 22$ although newer assay techniques using mass spectroscopy may not pose this problem. ${ }^{23}$ The upper limits of the laboratory ranges for UFC tend to be lower in the HPLC and LC-MS/MS techniques than in antibody-based assays.

\section{Overnight dexamethasone suppression test}

The test can be easily performed as an outpatient procedure if the patient can follow the required instructions. One milligram of dexamethasone is taken orally by the patient between 23:00 and 00:00, and serum cortisol is measured the next morning between 08:00 and 09:00. Cortisol level $<50 \mathrm{nmol} / \mathrm{L}$ is considered as the appropriate response after ODST in normally

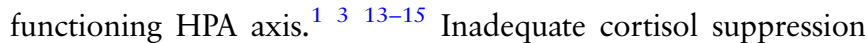
with higher cortisol levels has a sensitivity $>95 \%$ and a specificity of $80 \%$ for initial diagnosis of CS. ${ }^{3} 1314$ However, when the cut-off value $<140 \mathrm{nmol} / \mathrm{L}$ is used, the specificity for this test reaches $>95 \%,{ }^{13} 1524$ although sensitivity falls significantly.

A variety of drugs and clinical conditions can affect the bioavailability of dexamethasone, potentially interfering with

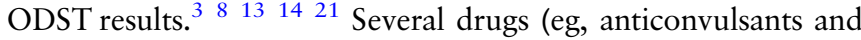
alcohol) induce hepatic CYP 3A4 enzyme and accelerate dexamethasone metabolism causing a false positive result. Other drugs may inhibit (eg, ritonavir and diltiazem) the enzyme increasing the serum levels. ${ }^{13}$ False positive ODST can occur in up to $50 \%$ of females on long-term oestrogen replacement such as the combined oral contraceptive pill or hormone replacement therapy because of its effect, which elevates CBG levels. ${ }^{13} 25$ Therefore, women should refrain from oestrogen-containing medications for at least six weeks prior to the test. ${ }^{12}{ }^{26}$ Valassi et $a l^{27}$ demonstrated a dexamethasone-suppressed cortisol level of $\leq 38 \mathrm{nmol} / \mathrm{L}$ in significantly higher number of cases not taking interfering medications that may alter dexamethasone metabolism compared with those who were taking these drugs (serotonin uptake inhibitors, lipid-lowering agents, calcium channel blockers, angiotensin II receptor antagonists, antipsychotics, proton pump inhibitors, antiarrhythmics, thiazolidinediones, $\beta$-adrenoceptor blockers, benzodiazepines and anticonvulsants).

Advanced stages of liver and renal diseases may also affect serum dexamethasone levels with false positive/negative ODST results. $^{13} 28$ Serum dexamethasone levels may vary even in normal individuals, and this may also interfere with the ODST result interpretation. ${ }^{13} 29$ Although the standard recommended dose for ODST is $1 \mathrm{mg}$ of dexamethasone, the use of $0.5 \mathrm{mg}$ dose has shown better sensitivity for the diagnosis of CS in Southeast Asian populations with lower body mass indices. ${ }^{30}$

Similar to the UFC measurement, variations in estimated serum cortisol levels may occur depending on the assay used. Although measurements were previously by colorimetric, spectroscopic or chromatographic methods, IAs with radioactive or non-isotopic labels are now commonly used by most laboratories. IAs can be easily automated with $>30$ different methods, which are available as manual kits and/or on automated platforms that use a radio immunoassay (RIA), an enzyme immunoassay (EIA), a fluorescence immunoassay (FIA) or a luminex immunoassay (LIA). For cortisol measurements, most laboratories now use automated IAs based on the competitive binding principle in which cortisol from clinical specimen and the exogenous labelled cortisol compete for binding sites on the anticortisol antibody that is detected by (chemi)luminescent signal generation. However, the between-assay agreements of these methods are often unsatisfactory. ${ }^{31}$

Most routine hospital laboratories use IAs while major reference laboratories use the LC-MS/MS method because chromatographic methods using mass spectrometry are more specific and accurate. The LC-MS/MS method detects different cortisol molecules based on their mass-to-charge ratio, without the interference from synthetic steroid molecules that may cross-react with cortisol antibodies in IAs because of structural similarity. ${ }^{31}$ Although this method has become a more popular technique recently, it is costlier and has limited availability.

\section{Low-dose dexamethasone suppression test}

Similar to ODST, this test can be performed as an outpatient procedure for a well-instructed patient. This test may be more specific in excluding conditions that may be associated with 
PCS. ${ }^{8} 13$ Dexamethasone is given in doses of $0.5 \mathrm{mg}$ at sixhourly intervals for 48 hours starting at 09:00 on day 1, and serum cortisol measurement is performed at 09:00 on day 3, 6 hours after the last dexamethasone dose. For patients weighing $<40 \mathrm{~kg}$, the dose should be adjusted to $30 \mu \mathrm{g} / \mathrm{kg} /$ day (in divided doses). ${ }^{32}$ Suppression of cortisol $<50 \mathrm{nmol} / \mathrm{L}$ is regarded as the normal response in an individual without CS.

The reported sensitivity and specificity of LDDST in diagnosing CS were $100 \%$ and $88 \%$, respectively, ${ }^{33}$ with a positive predictive value (PPV) of $92 \%$ and negative predictive value (NPV) of $89 \%$, respectively. ${ }^{8}$ Confounding factors that may interfere with ODST described above should be considered in the interpretation of the LDDST results.

\section{Late night salivary cortisol}

In a healthy individual with normal undisturbed sleep pattern, plasma cortisol starts rising between 03:00 and 04:00, peaks between 07:00 and 09:00, and then falls steadily with the lowest level being at midnight. This circadian rhythm of cortisol secretion may be altered in patients with CS who do not show the late night cortisol nadir that forms the physiological basis for measurement of LNSC. This is a very sensitive test that has been used by endocrinologists over the past two decades for biochemical screening and diagnosis of CS. ${ }^{13}$ 34-36 There is a steady equilibrium of serum-free cortisol with salivary cortisol that is unaffected by the rate of saliva production, and the test measures free cortisol fraction of unbound cortisol in the plasma. Serum cortisol fluctuations are reflected in the measured salivary cortisol levels within a short period of time. ${ }^{13}$ For this reason, salivary cortisol measurement may be feasible instead of serum cortisol assay after ODST, ${ }^{28}$ although this approach is not adequately validated.

Sample collection of saliva for LNSC is usually performed between 23:00 and 00:00 by asking the patient to chew a cylindrical cotton swab (salivette) for 2-3 min and keeping the sample in a plastic tube at $2-8^{\circ} \mathrm{C}$ overnight in order to be processed the following morning. Clinicians should carefully explain the procedure to the patient and should ensure timing of sample collection has been accurate before interpreting the result. LNSC should be performed twice (ideally in a row) to improve the sensitivity and specificity. Two samples are ideal to avoid false negativity in occasional circumstances because of the rhythmic nature of cortisol hypersecretion in some CS cases and the likelihood of inadequate quantity of saliva in the collected specimens. Although assay technique may have an effect on the diagnostic performance of the test, most studies reported sensitivity and specificity of LNSC $>95 \%$ in diagnosing CS. ${ }^{13}$ 34-37 However, it should be kept in mind that uncertainties exist in the influence of age, gender, coexistent medical conditions (eg, depression, critical illness, etc) on the diagnostic utility of LNSC. $^{13}$

Initial methods used for salivary cortisol measurements were similar to those used for serum and urinary cortisol assays. These were modified later to improve the performance of LNSC, in which small volumes of saliva were used and the low levels of cortisol in saliva were in comparison to the serum cortisol levels. RIA, ELISA, platform IAs and LC-MS/MS are the standard methods used for the test. ${ }^{36}$ Advantages of IAs are that they detect other cortisol metabolites, are easy to run and perform, are relatively inexpensive and require smaller volumes of saliva. Newer IAs are capable of detecting low cortisol levels at small volumes of saliva used for LNSC. Similar to the measurements of UFC and serum cortisol, chromatographic methods using mass spectrometry may give better diagnostic yield in LNSC. ${ }^{38} 39$ However, spectroscopic methods require higher volumes of saliva $(100-250 \mu \mathrm{L}$ by extraction), are costlier and the cut-off cortisol levels are also higher $(0.3-2.0 \mathrm{nmol} /$ L). ${ }^{39}$

The advantages of LNSC measurement for CS screening and diagnosis are the ease of home specimen collection from a wellinstructed patient, lack of interference from factors such as concurrent medication use that affect serum total and free cortisol levels, high sensitivity and specificity of the test, thermal stability at room temperature that helps easy transport of the specimen to laboratories and better diagnostic sensitivity even in mild cases of CS. ${ }^{3} 13$ 35-37 The major disadvantages are higher false positive rates in the elderly, hypertensives, diabetics and when IAs are used that cross-react with cortisone (formed from cortisol by $11 \beta$-hydroxysteroid dehydrogenase type 2 of salivary glands). ${ }^{3}$

\section{EXCLUSION OF OTHER HYPERCORTISOLISM STATES}

A positive biochemical screening for CS requires a thorough re-evaluation of both the patient and the clinical scenario to exclude other hypercortisolism states such as SCS, PCS and iatrogenic CS from exogenous steroid use. This will help to avoid subsequent expensive investigations to confirm the diagnosis of CS.

SCS is defined as a state of inappropriate cortisol overproduction that may be associated with alterations in the HPA axis, without the overt manifestations of classic CS such as striae, adiposity, proximal myopathy and metabolic abnormalities. ${ }^{40}$ The disease may be present in $5-30 \%$ of patients with adrenal incidentalomas, which is found in $4-7 \%$ of the adult population. ${ }^{9}$ Therefore, the estimated prevalence of the disease is about 0.2$2 \%$ in the adult population. Disagreement still remains among endocrine professional bodies on the diagnostic cut-offs for biochemical screening tests for SCS. ${ }^{9}{ }^{41}$ Although alteration of cortisol circadian rhythm assessed by both LNSC or serum cortisol measurements may provide reasonable diagnostic accuracy, the sensitivity and specificity of these assays may be inadequate as a single screening test. ${ }^{9}$ Therefore, higher cut-offs of individual tests and/or multiple tests may need to be developed for initial screening of SCS. An initial evaluation by ODST may be the best approach to patients with SCS and adrenal incidentalomas as suggested by multiple studies and consensus. ${ }^{42-46}$

PCS can prove to be a diagnostic challenge as many of the patients may have clinical features of CS, along with deceptive initial screening test results. Inadequate suppression of serum cortisol on ODST and an elevated UFC level were observed in $85 \%$ and $40 \%$ of subjects respectively with PCS, which make these tests less useful as initial screening tools. ${ }^{8}$ However, LNSC and midnight serum cortisol results (MserC) were observed to have a much better diagnostic accuracy in this study. Receiver operating characteristic curve analysis for LNSC using a cortisol cut-off value of $9.3 \mathrm{nmol} / \mathrm{L}$ gave a sensitivity of $100 \%$, specificity of $83 \%$, PPV of $94 \%$ and a NPV of $100 \%$ for distinguishing CS from PCS. ${ }^{8}$ Similarly, a single MserC concentration of $>243 \mathrm{nmol} / \mathrm{L}$ had a PPV of $98 \%$ with an NPV of $95 \%$ in differentiating CS from PCS.

Desamino-D-arginine vasopressin (Desmopressin; dDAVP) test is another important diagnostic tool for distinguishing patients with CD from PCS. ${ }^{47-50}$ The test is performed by serial measurements of serum cortisol and ACTH at baseline and after intravenous injection of $10 \mu \mathrm{g}$ of dDAVP. A basal serum cortisol $>331 \mathrm{nmol} / \mathrm{L}$ and an absolute ACTH increment $>4 \mathrm{pmol} / \mathrm{L}$ following dDAVP injection yielded a sensitivity of $90.3 \%$ and specificity of $91.5 \%$ in differentiating CD from PCS. ${ }^{47}$ 
A $4 \mathrm{mg}$ intravenous dexamethasone infusion that is run from 23:00 to 03:00 along with a measurement of serum ACTH levels later at 08:00 was recently reported to be useful to distinguish between patients with $\mathrm{CD}$ and PCS. ${ }^{51} \mathrm{~A}$ cut-off serum ACTH level of $14.8 \mathrm{ng} / \mathrm{L}(3.26 \mathrm{pmol} / \mathrm{L})$ has shown a sensitivity of $100 \%$ and specificity of $83.3 \%$ in distinguishing CD from PCS.

Pregnancy is a state of physical and psychological stress associated with a complex neuroendocrine immune response that results in activation of HPA axis, stimulation of innate immunity and release of pro-inflammatory cytokines. ${ }^{52}$ Increased production of CRH and ACTH occurs in pregnancy with a resultant rise in plasma cortisol levels. ${ }^{53}$ However, high levels of CBG because of hyper-oestrogenaemia of pregnancy help in maintaining the bioavailable free cortisol levels near the normal physiological limits. Pregnancy is also a type of low-grade chronic inflammatory state that downregulates response of glucocorticoid receptors to cortisol binding, a state of glucocorticoid resistance, and the hypercortisolemia of pregnancy may interfere with various biochemical tests for CS diagnosis.

Iatrogenic CS can occur because of exogenous administration of glucocorticoids for a number of autoimmune or inflammatory disorders. Occasionally, the presence of steroid molecules in some everyday food items, dietary supplements and indigenous herbal medicinal preparations may result in iatrogenic CS when consumed regularly on a long-term basis. Suppression of the HPA axis, and even adrenal atrophy, can result from these situations. Phenotypic features of CS with elevated (if patient is on prednisolone or hydrocortisone) or low serum cortisol (as several synthetic steroid molecules are not detected by routine cortisol assays) and suppressed ACTH levels are characteristic of this condition. Physiological doses of glucocorticoids, both in the topical or inhaled preparations, may also result in iatrogenic CS during concomitant co-administration of some medications in the same patient due to drug interference with the hepatic steroid metabolic pathyways. ${ }^{54}$ Therefore, a thorough patient history should always be taken before further investigating cases with suspected CS.

\section{CONFIRMATORY BIOCHEMICAL TESTS}

Following the initial positive screening tests for the possibility of CS, clinicians should proceed with further diagnostic investigations for biochemical characterisation of the disease. This should then be followed by anatomical localisation studies for subsequent management.

If two of the initial screening tests are positive with a high pre-test probability of the disease, diagnosis of CS is almost certain in that patient. ${ }^{113}$ Simultaneous or sequential screening tests with or without other tests could help in establishing the biochemical confirmation of CS. Additional biochemical tests may still be occasionally required to exclude PCS. LDDST combined with a CRH stimulation test (performed by an intravenous administration of $1 \mu \mathrm{g} / \mathrm{kg}$ of $\mathrm{CRH}$ two hours following the last dexamethasone dose and cortisol measurement $15 \mathrm{~min}$ afterwards) or a late MserC assay may be necessary to confirm the diagnosis of CS in some patients. ${ }^{13} 54$

The subsequent step after biochemical confirmation is to identify the underlying cause of the excess cortisol production. A baseline serum ACTH-level estimation may provide information regarding the abnormal source/stimulant for cortisol excess. Further discussion about the distinguishing laboratory features between different types of CS can be found in the next section.

Laboratory measurements of plasma ACTH level use IAs based on the 'sandwich' principle in which two antibodies recognise different ACTH epitopes. ${ }^{55}$ Enzyme-based or chemiluminescent labels are used in these assays for diagnostic purposes. The ideal specimen for ACTH assay is EDTA plasma collected and sent to the laboratory on ice as the hormone is heat-labile (proteolytic degradation at higher temperatures). Both manual and automated IAs from different manufacturers are commercially available for ACTH estimation, with variable lab reference ranges depending on the methodology used.

\section{DIFFERENTIAL DIAGNOSIS OF CONFIRMED HYPERCORTISOLISM}

Pathophysiologically, the disease can be broadly classified into ACTH-dependent and ACTH-independent CS. About 70-80\% of CS cases belong to the former category and $20-30 \%$ to the latter. ${ }^{1}$ Pathophysiological characterisation by initial biochemical testing is important for appropriate localisation studies to plan management of the disease. A suppressed serum ACTH level of $<2.2 \mathrm{pmol} / \mathrm{L}$ with overt hypercortisolism usually indicates an adrenal pathology as the cause of CS. ${ }^{113}{ }^{54}$ Serum ACTH level $>4.4 \mathrm{pmol} / \mathrm{L}$ may suggest an ACTH-dependent CS from extraadrenal disease, often from pituitary pathology (CD). Intermediate values of serum ACTH between 2.2 and $4.4 \mathrm{pmol} /$ $\mathrm{L}$ would necessitate further evaluation with a $\mathrm{CRH}$ stimulation test. $^{15456}$

\section{ACTH-independent CS}

In total, 20-30\% of CS cases result from adrenal pathology mostly from unilateral disease and less commonly from bilateral gland involvement. ${ }^{1}$ Unilateral adrenal adenomas and less often carcinomas account for the majority of cases of adrenal CS. Bilateral disease results from multiple adenomas, micronodular or macronodular hyperplasia, and rarely from McCune-Albright syndrome and carcinomas. ${ }^{1}$

Suppressed ACTH levels (<2.2 pmol/L), with raised serum/ urinary cortisol levels non-suppressible on dexamethasone challenge, usually indicate adrenal CS. Elevated androgen levels and virilisation may be seen in CS resulting from adrenal carcinoma because of sex hormone co-secretion. ${ }^{1} 57$ Raised levels of steroid precursors along with androgen and mineralocorticoid hypersecretion may be seen in bilateral macronodular adrenal hyperplasia. ${ }^{158}$ A paradoxical rise in UFC levels may be seen in the rare form of adrenal CS-primary pigmented adrenocortical nodular disease-after dexamethasone suppression tests. ${ }^{1}$ Once the diagnosis of adrenal CS is established biochemically, the clinician can proceed with adrenal imaging to identify the causative pathology.

\section{ACTH-dependent CS}

In total, $60-70 \%$ of these cases result from CD related to a corticotroph adenoma and very rarely from corticotroph hyperplasia that causes ACTH hypersecretion. ${ }^{1}$ Higher baseline ACTH levels (usually $>4.4 \mathrm{pmol} / \mathrm{L}$ ) usually suggest ACTH-dependent disease, although in some cases of CD the levels may be inappropriately in the normal range (hypercortisolism normally suppresses ACTH). ${ }^{54}$ Uncommonly, some cases of ACTH-independent CS from mild adrenal CS may not suppress ACTH $<2.2 \mathrm{pmol} / \mathrm{L}$ as observed in some cases of CD. In such cases, a CRH stimulation test or dDAVP stimulation test becomes necessary. Abnormal corticotroph cells in the pituitary adenoma causing mild forms of CD will still respond to the tests by an increase in ACTH production, whereas the chronically suppressed normal corticotroph cells in cases with adrenal CS will not respond. ${ }^{54}$ 


\section{Ectopic ACTH-driven CS}

An extraordinarily high serum ACTH levels (>110 pmol/L) would usually indicate an ectopic ACTH production as the causative pathology. ${ }^{5459}{ }^{60}$ Profound hypercortisolism, shorter duration of clinical illness and more severe hypokalaemia are seen in many of these cases compared to patients with CD. ${ }^{61}$ Detection of raised levels of neuroendocrine hormones and chemical mediators such as calcitonin, gastrin, chromogranin A and 5-hydroxyindoleacetic acid in biological specimens (serum/ urine) from patients with CS may suggest ectopic ACTH-driven CS as the majority of these cases result from neuroendocrine tumours (NETs).

Although high-dose dexamethasone suppression test (HDDST) is not favoured by many endocrinologists, some centres still perform the test to distinguish between $\mathrm{CD}$ and ectopic ACTH-driven CS. HDDST relies upon the fact that pituitary corticotroph tumour cells retain a degree of negative feedback response to glucocorticoids, unlike extra-pituitary tumours. ${ }^{2}{ }^{63}$ In the standard/classic test, serum cortisol or UFC is measured after oral dexamethasone $2 \mathrm{mg}$ administration every six hours for 2 days (total eight doses). Alternatively, $8 \mathrm{mg}$ dexamethasone can be administered orally at 23:00 with a subsequent serum cortisol measurement taken at 08:00 on the following day. A baseline cortisol measurement is performed in both these methods; a suppression of cortisol $>50 \%$ compared with the baseline value would indicate a positive test result, which is suggestive of CS with a pituitary origin. ${ }^{1} 6263$ However, about one-third of patients with ectopic ACTH-driven CS may also show cortisol suppression at these levels. ${ }^{61-63}$ Using logistic regression models from a large series of cases, Aron $e t$ al $^{61}$ previously showed that HDDST adds little to the differential diagnosis of ACTH-dependent CS, especially after taking other clinical information into account. However, serum cortisol suppression with a cut-off value of $<33.3 \%$ after HDDST has shown a sensitivity and specificity of $95.8 \%$ and $90.6 \%$ respectively in a recent study reported from Korea. ${ }^{64}$

\section{INVESTIGATIONS FOR LOCALISATION ACTH-dependent disease}

If pituitary cause (CD) is suspected, a dedicated pituitary imaging study should be performed as the next step. A contrast-enhanced MRI of the pituitary is the preferred imaging modality. MRI identifies the pituitary tumours in $>50 \%$ of patients with $\mathrm{CD}$, and the majority $(80-90 \%)$ of these lesions are microadenomas $\left(<10 \mathrm{~mm}\right.$ size). ${ }^{1}{ }^{13} 606365$ Lesions $<6 \mathrm{~mm}$ with unknown biochemical significance are seen in about $10 \%$ of the general adult population, and therefore, microadenomas $<6 \mathrm{~mm}$ may not be diagnostic of CD. ${ }^{1}$ Newer MRI techniques such as methionine-positron emission tomography (MET-PET) and flouro-deoxy-glucose-PET (FDG-PET) using superconductive MRI sequences $(3.0 \mathrm{~T})$ demonstrated improved accuracy rates of up to $100 \%$ and $73 \%$, respectively, compared with conventional MRI that showed accuracy rates $<50 \%{ }^{66}$

When biochemical and anatomical diagnostic investigations do not reliably distinguish between $\mathrm{CD}$ and ectopic ACTH-driven CS, a bilateral inferior petrosal sinus sampling (BIPSS) is often considered to aid diagnostic confirmation and management plan. ${ }^{1606366} 67$ Although this is an invasive test that requires interventional radiology expertise in a specialised centre, BIPSS remains a key investigation that is necessary in a proportion of CS cases in whom biochemical and anatomical localisation studies are inconclusive to establish the diagnosis. A central to peripheral ACTH gradient $>2$ that raises to $>3$ after
$\mathrm{CRH}$ stimulation during BIPSS procedure is diagnostic of $\mathrm{CD}$ with a sensitivity and specificity of approximately $95 \% .{ }^{1}{ }^{63} 67$ Measurement of prolactin levels in plasma collected from BIPSS sample improves the sensitivity and helps exclusion of ectopic source of ACTH with higher accuracy ${ }^{68}{ }^{69}$ In occasional cases, CD may occur in patients with empty sella syndrome, and localisation study with BIPSS may help the cure of CD by surgical resection in such cases. ${ }^{70}$

Localisation studies with the use of CT, MRI and PET imaging modalities can aid the diagnosis of ectopic ACTH-driven CS in the majority of cases. NETs of varying types can produce ACTH or CRH, which may account for up to $5-10 \%$ of CS. ${ }^{1}$ Many of these tumours may be small and be elusive to imaging modalities, rendering localisation studies a diagnostic challenge. Recent studies have shown a superiority of somatostatin analogue-based PET imaging over CT and MRI scans for localisation studies of these NETs. ${ }^{171}{ }^{72}$ For example, scintigraphy with ${ }^{68}$ Gallium-SSTR-PET/CT was reported to have $100 \%$ sensitivity in detecting NET with ACTH or CRH production in a recent study. ${ }^{72}$ However, limited availability and technical difficulties restrict the use of these newer investigative modalities in routine clinical practice.

\section{ACTH-independent disease}

An initial adrenal imaging modality such as CT or MRI scan is necessary for localisation of a pathology such as adrenal adenoma or cancer in patients with ACTH-independent disease. Adrenal venous sampling (AVS) is an invasive radiological localisation study performed by comparing the measured plasma cortisol from the right and left adrenal veins. This may be necessary to differentiate the adrenal gland that is responsible for cortisol overproduction, prior to further surgical/medical management in some patients. ${ }^{73}$ However, this approach has not been adequately explored, unlike the BIPSS.
Box 1 Genetic disorders or gene defects associated with Cushing's syndrome (CS)

Cushing's disease associated with genetic disease

- Multiple endocrine neoplasia (MEN-1) syndrome

- MEN-4 syndrome

- Mutations in aryl-hydrocarbon receptor-interacting protein, cyclin-dependent kinase inhibitor, USP8, CDKN1B (p27Kip1), CDKN2C (p18INK4c), cyclin E (CCNE), EGFR, CMPtk and LAPTM4B genes.

Ectopic adrenocorticotropin-driven CS associated with genetic disease

- MEN-1 syndrome

- RET gene mutations

Adrenal CS associated with genetic disease

- Adrenal adenomas - defects in PRKACA, CTNNB1, GCPR, GNAS1 and PRKAR1A genes

- Adrenal hyperplasia-defects in ARMC5, MEN1, FH, GNAS1, PDE11A, PDE8B, MC2R, PRKACA, GPCR and PRKAR1A genes

- Adrenal cancer-defects in P53 (Li-Fraumeni syndrome), GNAS, MEN-1, IGF-II, H-19, CDKI (Beckwith-Wiedemann syndrome) and adenomatous polyposis colii genes 


\section{GENETIC TESTING}

Although most cases of CS are sporadic in nature, somatic and germline mutations are identified in some cases. Several genetic abnormalities associated with pituitary, adrenal and ectopic ACTH-driven CS are described as shown in box $1 .{ }^{174}$ Genetic testing may be necessary in patients with CS when multiple members of a family present with endocrine tumours as in multiple endocrine neoplasia 1 (MEN1) syndrome. More detailed discussion of these disorders and related diagnostic investigative methodologies can be found in other literature. ${ }^{174} 75$ When clinically suspected, appropriate testing should be arranged in collaboration with a clinical geneticist in such cases.

\section{CYCLICAL CS}

Cyclical CS results from rhythmic alterations in the cortisol production with fluctuations in both clinical and laboratory results. Diagnosis of cyclical CS requires demonstration of at least three peak levels and two trough levels in cortisol production on prolonged testing. 'Shoulders' in cortisol level (cortisol values above the normal baseline) can be seen in these patients before and after the peaks, indicating that cortisol levels were rising over a few days. Although more commonly seen in patients with CD, cyclical CS can also be encountered in patients with adrenal CS and ectopic ACTH-driven CS. ${ }^{6}$ Cyclicity has been reported in up to $15 \%$ of cases with $\mathrm{CD}$ in a large series. ${ }^{76}$

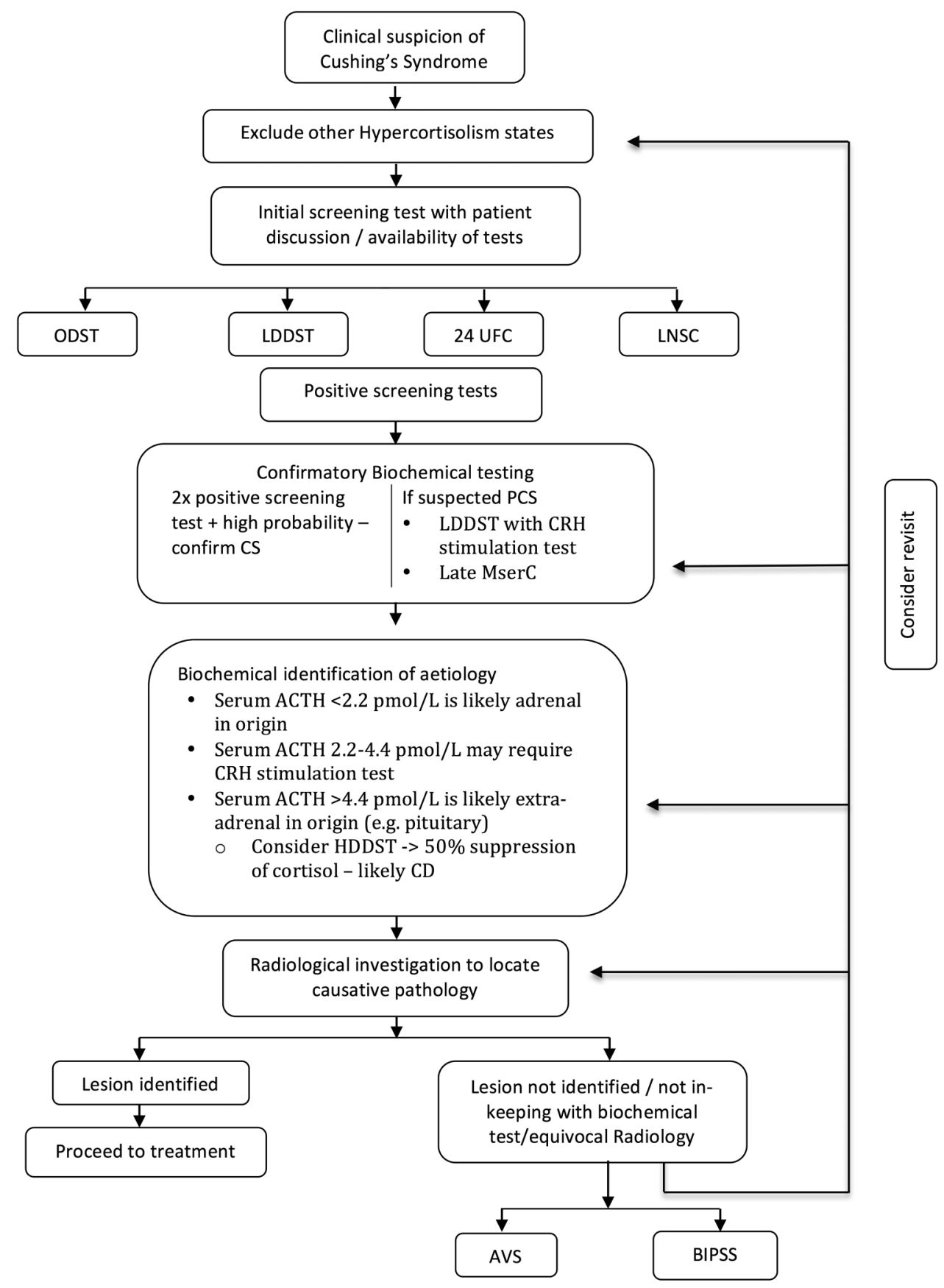

Figure 1 Cushing's syndrome: a diagnostic algorithm. ACTH, adrenocorticotropin; AVS, adrenal vein sampling; BIPSS, bilateral inferior petrosal sinus sampling; $C D$, Cushing's disease; $C R H$, corticotropin-releasing hormone; $C S$, Cushing's syndrome; HDDST, high-dose dexamethasone suppression test; LDDST, low-dose dexamethasone suppression test; LNSC, late night salivary cortisol; MserC, midnight serum cortisol; ODST, overnight dexamethasone suppression test; PCS, pseudo-Cushing's syndrome; UFC, urinary-free cortisol. 
Diagnostic evaluation for cyclical CS is often difficult and time consuming. Prolonged testing over a 28 -day period is recommended for suspected cases. ${ }^{67}$ Testing may become necessary in patients with a discordant biochemical profile on evaluation, a paradoxical response to DST, a suggestive clinical picture with normal/slightly abnormal laboratory findings, and in the recurrence of CS after surgery with normal test results. An abnormal early morning urine cortisol-to-creatinine ratio from a 28 consecutive day collection is diagnostic in the majority of cases. Occasional patients with cyclical CS with prolonged cycling intervals can be missed if this strategy is used..$^{77}$ Nocturnal salivary cortisol estimation over a 28 -day period corresponded well with urinary cortisol-to-creatinine ratio in a recent study and can be used as an alternative test for diagnostic evaluation of patients with cyclical CS. ${ }^{7}$ In patients with prolonged cycling intervals, extended testing for months or even years may be required to reach the diagnosis. ${ }^{36} 78$

\section{EVALUATION ALGORITHM}

An algorithm for diagnostic evaluation of patients with suspected CS is shown in figure 1 .

\section{AREAS OF UNCERTAINTY}

The influence of age, gender and various medical conditions such as depression and critical illness to the diagnostic performance of LNSC as a screening tool is not well known. Confounding factors such as poor acquisition techniques and contamination may interfere with diagnostic utility of this test. ${ }^{79}$ Sensitivity and specificity have recently been reported to be low in patients with SCS in a small series. ${ }^{80}$ These factors warrant further validation of LNSC in large-scale controlled studies to clear this uncertainty.

Although multiple screening tests as discussed above can reliably exclude PCS in most patients, some cases pose significant challenge to clinicians. Patients with obesity-related hypertension, resistant to conventional treatment, have a relatively high prevalence of SCS picture, and excluding hypercortisolism can prove to be extremely difficult. ${ }^{81}$ One author suggested that routine screening for CS has not proven to be a worthwhile nor a cost-effective evaluation method in patients with morbid obesity, hypertension or type 2 diabetes as the prevalence of CS in such patients is thought to be $<1 \%{ }^{82}$ Although screening young patients who have features of CS with hypertension and type 2 diabetes was suggested, there is no clear consensus about the optimal screening strategies for patients with PCS.

HDDST is still being performed in some centres for CS diagnostic evaluation. However, there is much dispute about the utility of this test. Although newer techniques such as MET-PET, FDG-PET and ${ }^{68}$ Gallium-SSTR-PET/CT are emerging as promising tools, the ideal imaging method for localisation in patients with difficult to diagnose extra-adrenal CS still remains uncertain. Similarly, the optimal duration of screening studies necessary for diagnostic evaluation of patients with suspected cyclical $\mathrm{CS}$ is not clear. The consensus recommendation adopted by most centres is by using a 28 -day screening period. ${ }^{6}{ }^{7}$ In some patients, the cycles can be more prolonged, creating a diagnostic challenge. $^{7} 78$

\section{CONCLUSIONS}

Diagnostic evaluation of patients with suspected CS remains challenging despite many years of research and published case series. The currently recommended standard screening tests have satisfactory sensitivity and specificity in case detection when two or more of these tests show positive results.
Clinicians should exclude other conditions with hypercortisolism phenotype prior to pursuing further localisation studies in the respective patients, although routine screening is not deemed necessary in most cases of PCS with a low pre-test probability. Diagnostic evaluation of cyclical CS and SCS can be both challenging and time consuming.

Localisation studies are often expensive and sometimes inconclusive. Although not time tested due to the recent development, the uses of newer imaging techniques such as MET-PET, FDG-PET and ${ }^{68}$ Gallium-SSTR-PET/CT are emerging as useful tools in localisation studies. Genetic testing may be considered if clinical picture suggests an inherited abnormality causing the disease. Confounding factors in optimal diagnostic performance of LNSC, identification of people with PCS who need clinical and diagnostic evaluation for CS, the role of HDDST in the CS diagnostic algorithm and the ideal imaging strategy for difficult to diagnose extra-adrenal CS are some of the areas of uncertainties in diagnostic evaluation of patients with CS that require further research.

\section{Take home messages}

- Two measurements of urinary free cortisol, two measurements of late night salivary cortisol, $1 \mathrm{mg}$ overnight dexamethasone suppression test and low dose dexamethasone suppression test are the initial screening biochemical tests for suspected cases of Cushing's syndrome.

- An overnight dexamethasone suppression test is the preferred screening method for patients with suspected subclinical Cushing's syndrome resulting from adrenal incidentalomas.

- Two or more positive initial screening tests in a patient with high pretest probability of the disease confirms the biochemical diagnosis of Cushing's syndrome.

- A baseline plasma adreonocorticotropin (ACTH) level measurement helps identification of the cause of hypercortisolism in most of the biochemically confirmed cases of Cushing's syndrome.

- Anatomical localisation of the cause of Cushing's syndrome involves imaging modalities such as contrast MRI of the pituitary (Cushing's disease), CT scan/ MRI scan of the adrenals (adrenal Cushing's), and CT/ PET scans of the thorax, abdomen and pelvis (ectopic ACTH-driven Cushing's).

\section{Handling editor Tahir Pillay}

Contributors JMP and $\mathrm{CH}$ contributed equally to the preparation of the manuscript. All authors contributed to literature search and final editing of the manuscript in the present form. A new author, a laboratory scientist, is added in the revision as suggested by the reviewer considering his substantial contribution to the paper revision.

Competing interests None declared.

Provenance and peer review Commissioned; externally peer reviewed.

\section{REFERENCES}

1 Lacroix A, Feelders RA, Stratakis CA, et al. Cushing's syndrome. Lancet 2015;386:913-27.

2 Ferraù $F$, Korbonits M. Metabolic comorbidities in Cushing's syndrome. Eur J Endocrinol 2015;173:M133-57.

3 Nieman LK. Cushing's syndrome: update on signs, symptoms and biochemical screening. Eur J Endocrinol 2015;173:M33-8.

4 Graversen D, Vestergaard P, Stochholm K, et al. Mortality in Cushing's syndrome: a systematic review and meta-analysis. Eur J Intern Med 2012;23:278-82. 
5 Clayton RN, Raskauskiene D, Reulen RC, et al. Mortality and morbidity in Cushing's disease over 50 years in Stoke-on-Trent, UK: audit and meta-analysis of literature. J Clin Endocrinol Metab 2011;96:632-42.

6 Atkinson B, Mullan KR. What is the best approach to suspected cyclical Cushing syndrome? Strategies for managing Cushing's syndrome with variable laboratory data. Clin Endocrinol (Oxf) 2011;75:27-30.

7 Graham UM, Hunter SJ, McDonnell M, et al. A comparison of the use of urinary cortisol to creatinine ratios and nocturnal salivary cortisol in the evaluation of cyclicity in patients with Cushing's syndrome. J Clin Endocrinol Metab 2013;98: E72-6.

8 Alwani RA, Schmit Jongbloed LW, de Jong FH, et al. Differentiating between Cushing's disease and pseudo-Cushing's syndrome: comparison of four tests. Eur J Endocrinol 2014;170:477-86.

9 Di Dalmazi G, Pasquali R, Beuschlein F, et al. Subclinical hypercortisolism: a state, a syndrome, or a disease? Eur J Endocrinol 2015;173:M61-71.

10 Chiodini I. Clinical review: diagnosis and treatment of subclinical hypercortisolism. J Clin Endocrinol Metab 2011;96:1223-36.

11 Broder MS, Chang E, Cherepanov D, et al. Identification of potential markers for Cushing disease. Endocr Pract 2016;22:567-74.

12 Hopkins RL, Leinung MC. Exogenous Cushing's syndrome and glucocorticoid withdrawal. Endocrinol Metab Clin North Am 2005:34:371-84, ix.

13 Nieman LK, Biller BM, Findling JW, et al. The diagnosis of Cushing's syndrome: an Endocrine Society Clinical Practice Guideline. J Clin Endocrinol Metab 2008:93:1526-40.

14 Elamin MB, Murad MH, Mullan R, et al. Accuracy of diagnostic tests for Cushing syndrome: a systematic review and meta-analyses. J Clin Endocrinol Metab 2008;93:1553-62.

15 Ceccato F, Barbot M, Zilio M, et al. Screening tests for Cushing's syndrome: urinary free cortisol role measured by LC-MS/MS. J Clin Endocrinol Metab 2015;100:3856-61.

16 Aranda G, Careaga M, Hanzu FA, et al. Accuracy of immunoassay and mass spectrometry urinary free cortisol in the diagnosis of Cushing's syndrome. Pituitary 2016:19:496-502.

17 Chan KC, Lit LC, Law EL, et al. Diminished urinary free cortisol excretion in patients with moderate and severe renal impairment. Clin Chem 2004;50:757-9.

18 Mericq MV, Cutler GB Jr. High fluid intake increases urine free cortisol excretion in normal subjects. J Clin Endocrinol Metab 1998:83:682-4.

19 Petersenn S, Newell-Price J, Findling JW, et al. Pasireotide B2305 Study Group. High variability in baseline urinary free cortisol values in patients with Cushing's disease. Clin Endocrinol (Oxf) 2014;80:261-9.

20 Guarnotta V, Amato MC, Pivonello $R$, et al. The degree of urinary hypercortisolism is not correlated with the severity of cushing's syndrome. Endocrine. Published Online First: 10 Mar 2016. doi:10.1007/s12020-016-0914-9.

21 Prague JK, May S, Whitelaw BC. Cushing's syndrome. BMJ 2013;346:f945.

22 Turpeinen U, Markkanen $\mathrm{H}$, Välimäki M, et al. Determination of urinary free cortisol by HPLC. Clin Chem 1997:43:1386-91.

23 Meikle AW, Findling J, Kushnir MM, et al. Pseudo-Cushing syndrome caused by fenofibrate interference with urinary cortisol assayed by high-performance liquid chromatography. J Clin Endocrinol Metab 2003;88:3521-4.

24 Tateishi $Y$, Kouyama R, Mihara M, et al. Evaluation of salivary cortisol measurements for the diagnosis of subclinical Cushing's syndrome. Endocr J 2012;59:283-9.

25 Nickelsen T, Lissner W, Schöffling K. The dexamethasone suppression test and long-term contraceptive treatment: measurement of ACTH or salivary cortisol does not improve the reliability of the test. Exp Clin Endocrinol 1989:94:275-80.

26 Qureshi AC, Bahri A, Breen LA, et al. The influence of the route of oestrogen administration on serum levels of cortisol-binding globulin and total cortisol. Clin Endocrinol (Oxf) 2007;66:632-5.

27 Valassi $\mathrm{E}$, Swearingen $\mathrm{B}$, Lee $\mathrm{H}$, et al. Concomitant medication use can confound interpretation of the combined dexamethasone-corticotropin releasing hormone test in Cushing's syndrome. J Clin Endocrinol Metab 2009;94:4851-9.

28 Cardoso EM, Arregger AL, Budd D, et al. Dynamics of salivary cortisol in chronic kidney disease patients at stages 1 through 4. Clin Endocrinol (Oxf) 2016:85:313-19.

29 Meikle AW. Dexamethasone suppression tests: usefulness of simultaneous measurement of plasma cortisol and dexamethasone. Clin Endocrinol (Oxf) 1982;16:401-8.

30 Kageyama K, Oki Y, Sakihara $\mathrm{S}$, et al. Evaluation of the diagnostic criteria for Cushing's disease in Japan. Endocr J 2013;60:127-35.

31 Turpeinen U, Hämäläinen E. Determination of cortisol in serum, saliva and urine. Best Pract Res Clin Endocrinol Metab 2013;27:795-801.

32 Magiakou MA, Mastorakos G, Oldfield EH, et al. Cushing's syndrome in children and adolescents. Presentation, diagnosis, and therapy. N Engl J Med 1994;331:629-36.

33 Martin NM, Dhillo WS, Banerjee A, et al. Comparison of the dexamethasone-suppressed corticotropin-releasing hormone test and low-dose dexamethasone suppression test in the diagnosis of Cushing's syndrome. J Clin Endocrinol Metab 2006;91:2582-6.
34 Doi SA, Clark J, Russell AW. Concordance of the late night salivary cortisol in patients with Cushing's syndrome and elevated urine-free cortisol. Endocrine 2013;43:327-33

35 Carroll T, Raff $\mathrm{H}$, Findling JW. Late-night salivary cortisol for the diagnosis of Cushing syndrome: a meta-analysis. Endocr Pract 2009;15:335-42.

36 Raff $\mathrm{H}$. Utility of salivary cortisol measurements in Cushing's syndrome and adrenal insufficiency. J Clin Endocrinol Metab 2009;94:3647-55.

37 Elias PC, Martinez EZ, Barone BF, et al. Late-night salivary cortisol has a better performance than urinary free cortisol in the diagnosis of Cushing's syndrome. J Clin Endocrinol Metab 2014;99:2045-51.

38 Carroll T, Raff H, Findling JW. Late-night salivary cortisol measurement in the diagnosis of Cushing's syndrome. Nat Clin Pract Endocrinol Metab 2008:4:344-50.

39 Raff H. Cushing's syndrome: diagnosis and surveillance using salivary cortisol. Pituitary 2012;15:64-70.

40 Bancos I, Alahdab F, Crowley RK, et al. THERAPY OF ENDOCRINE DISEASE: improvement of cardiovascular risk factors after adrenalectomy in patients with adrenal tumors and Subclinical Cushing Syndrome: a systematic review and meta-analysis. Eur J Endocrinol 2016;175:R283-95.

41 Shen J, Sun M, Zhou B, et al. Nonconformity in the clinical practice guidelines for subclinical Cushing's syndrome: which guidelines are trustworthy? Eur J Endocrinol 2014; 171:421-31.

42 Masserini B, Morelli V, Bergamaschi S, et al. The limited role of midnight salivary cortisol levels in the diagnosis of subclinical hypercortisolism in patients with adrenal incidentaloma. Eur J Endocrinol 2009;160:87-92.

43 Deutschbein T, Unger N, Hinrichs J, et al. Late-night and low-dose dexamethasone-suppressed cortisol in saliva and serum for the diagnosis of cortisol-secreting adrenal adenomas. Eur J Endocrinol 2009;161:747-53.

44 Palmieri S, Morelli V, Polledri E, et al. The role of salivary cortisol measured by liquid chromatography-tandem mass spectrometry in the diagnosis of subclinical hypercortisolism. Eur J Endocrinol 2013;168:289-96.

45 Akehi $Y$, Kawate $\mathrm{H}$, Murase $\mathrm{K}$, et al. Proposed diagnostic criteria for subclinical Cushing's syndrome associated with adrenal incidentaloma. Endocr $J$ 2013:60:903-12

46 Fassnacht M, Arlt W, Bancos I, et al. Management of adrenal incidentalomas: European Society of Endocrinology Clinical Practice Guideline in collaboration with the European Network for the Study of Adrenal Tumors. Eur J Endocrinol 2016;175: G1-34.

47 Moro $M$, Putignano $\mathrm{P}$, Losa $\mathrm{M}$, et al. The desmopressin test in the differential diagnosis between Cushing's disease and pseudo-Cushing states. J Clin Endocrinol Metab 2000;85:3569-74.

48 Pecori Giraldi F, Pivonello R, Ambrogio AG, et al. The dexamethasone-suppressed corticotropin-releasing hormone stimulation test and the desmopressin test to distinguish Cushing's syndrome from pseudo-Cushing's states. Clin Endocrinol (Oxf) 2007:66:251-7.

49 Tirabassi G, Faloia E, Papa R, et al. Use of the desmopressin test in the differential diagnosis of pseudo-Cushing state from Cushing's disease. J Clin Endocrinol Metab 2010;95:1115-22.

50 Tamada D, Otsuki M, Kashine $S$, et al. Obstructive sleep apnea syndrome causes a pseudo-Cushing's state in Japanese obese patients with type 2 diabetes mellitus. Endocr J 2013:60:1289-94.

51 Nouvel M, Rabilloud M, Raverot V, et al. Performance of the 4-mg intravenous dexamethasone suppression test in differentiating Cushing disease from pseudo-Cushing syndrome. Ann Endocrinol (Paris) 2016;77:30-6.

52 Corwin EJ, Guo Y, Pajer K, et al. Immune dysregulation and glucocorticoid resistance in minority and low income pregnant women. Psychoneuroendocrinology 2013;38:1786-96.

53 Bronstein MD, Machado MC, Fragoso MC. Management of endocrine disease: management of pregnant patients with Cushing's syndrome. Eur J Endocrinol 2015;173:R85-91.

54 Raff H, Sharma ST, Nieman LK. Physiological basis for the etiology, diagnosis, and treatment of adrenal disorders: Cushing's syndrome, adrenal insufficiency, and congenital adrenal hyperplasia. Compr Physiol 2014;4:739-69.

55 Oliver RL, Davis JR, White A. Characterisation of ACTH related peptides in ectopic Cushing's syndrome. Pituitary 2003;6:119-26.

56 Boscaro M, Arnaldi G. Approach to the patient with possible Cushing's syndrome. J Clin Endocrinol Metab 2009:94:3121-31.

57 Bonfig W, Bittmann I, Bechtold S, et al. Virilising adrenocortical tumours in children. Eur J Pediatr 2003;162:623-8.

58 Espiard S, Drougat L, Libé R, et al. ARMC5 mutations in a large cohort of primary macronodular adrenal hyperplasia: clinical and functional consequences. I Clin Endocrinol Metab 2015;100:E926-35.

59 Raff $\mathrm{H}$, Carroll T. Cushing's syndrome: from physiological principles to diagnosis and clinical care. J Physiol (Lond) 2015:593:493-506.

60 Findling JW, Raff H. Cushing's Syndrome: important issues in diagnosis and management. J Clin Endocrinol Metab 2006;91:3746-53.

61 Aron DC, Raff H, Findling JW. Effectiveness versus efficacy: the limited value in clinical practice of high dose dexamethasone suppression testing in the differential 
diagnosis of adrenocorticotropin-dependent Cushing's syndrome. J Clin Endocrinol Metab 1997;82:1780-5.

62 Vilar L, Freitas Mda C, Faria M, et al. Pitfalls in the diagnosis of Cushing's syndrome. Arq Bras Endocrinol Metabol 2007:51:1207-16.

63 Machado MC, Fragoso MC, Moreira AC, et al. Recommendations of the Neuroendocrinology Department of the Brazilian Society of Endocrinology and Metabolism for the diagnosis of Cushing's disease in Brazil. Arch Endocrinol Metab 2016:60:267-86.

64 Hong AR, Kim JH, Hong ES, et al. Limited diagnostic utility of plasma adrenocorticotropic hormone for differentiation between adrenal Cushing syndrome and Cushing disease. Endocrinol Metab (Seoul) 2015;30:297-304.

65 Nieman LK, Biller BM, Findling JW, et al., Endocrine Society. Treatment of Cushing's syndrome: an Endocrine Society Clinical Practice Guideline. J Clin Endocrinol Metab 2015;100:2807-31.

66 Ikeda H, Abe T, Watanabe K. Usefulness of composite methionine-positron emission tomography/3.0-tesla magnetic resonance imaging to detect the localization and extent of early-stage Cushing adenoma. J Neurosurg 2010;112:750-5.

67 Page-Wilson G, Freda PU, Jacobs TP, et al. Clinical utility of plasma POMC and AgRP measurements in the differential diagnosis of ACTH-dependent Cushing's syndrome. J Clin Endocrinol Metab 2014;99:E1838-45.

68 Raff H. Cushing syndrome: update on testing. Endocrinol Metab Clin North Am 2015:44:43-50.

69 Mulligan GB, Faiman C, Gupta M, et al. Prolactin measurement during inferior petrosal sinus sampling improves the localization of pituitary adenomas in Cushing's disease. Clin Endocrinol (Oxf) 2012;77:268-74.

70 Mehta GU, Bakhtian KD, Oldfield EH. Effect of primary empty sella syndrome on pituitary surgery for Cushing's disease. J Neurosurg 2014;121:518-26.
71 Santhanam P, Taieb D, Giovanella L, et al. PET imaging in ectopic Cushing syndrome: a systematic review. Endocrine 2015;50:297-305.

72 Isidori AM, Sbardella E, Zatelli MC, et al. Conventional and nuclear medicine imaging in ectopic Cushing's syndrome: a systematic review. J Clin Endocrinol Metab 2015;100:3231-44.

73 Martins RG, Agrawal R, Berney DM, et al. Differential diagnosis of adrenocorticotropic hormone-independent Cushing syndrome: role of adrenal venous sampling. Endocr Pract 2012;18:e153-7.

74 Lodish M. Cushing's syndrome in childhood: update on genetics, treatment, and outcomes. Curr Opin Endocrinol Diabetes Obes 2015;22:48-54.

75 Yaneva M, Vandeva S, Zacharieva S, et al. Genetics of Cushing's syndrome. Neuroendocrinology 2010;92(Suppl 1):6-10.

76 Alexandraki KI, Kaltsas GA, Isidori AM, et al. The prevalence and characteristic features of cyclicity and variability in Cushing's disease. Eur I Endocrinol 2009;160:1011-18.

77 Mullan KR, Atkinson AB, Sheridan B. Cyclical Cushing's syndrome: an update. Curr Opin Endocrinol Diabetes Obes 2007;14:317-22.

78 Mosnier-Pudar $\mathrm{H}$, Thomopoulos P, Bertagna $\mathrm{X}$, et al. Long-distance and long-term follow-up of a patient with intermittent Cushing's disease by salivary cortisol measurements. Eur J Endocrinol 1995;133:313-16.

79 Bansal V, El Asmar N, Selman WR, et al. Pitfalls in the diagnosis and management of Cushing's syndrome. Neurosurg Focus 2015;38:E4.

80 Kuzu I, Zuhur SS, Demir N, et al. The diagnostic value of late-night salivary cortisol for diagnosis of subclinical Cushing's syndrome. Endokrynol Pol 2016;67:487-92.

81 Varughese AG, Nimkevych 0, Uwaifo Gl. Hypercortisolism in obesity-associated hypertension. Curr Hypertens Rep 2014;16:443.

82 Shimon I. Screening for Cushing's syndrome: is it worthwhile? Pituitary 2015;18:201-5. 